\title{
BMS-536924, an ATP-competitive IGF-I R/IR inhibitor, decreases viability and migration of temozolomide-resistant glioma cells in vitro and suppresses tumor growth in vivo
}

\author{
This article was published in the following Dove Press journal: \\ OncoTargets and Therapy \\ 2 April 2015 \\ Number of times this article has been viewed
}

\author{
Qiao Zhou \\ School of Public Affairs, University \\ of Science and Technology of China, \\ Hefei, Anhui, People's Republic \\ of China
}

Correspondence: Qiao Zhou School of Public Affairs, University of Science and Technology of China, 96 Jinzhai Road, Hefei, Anhui, 230026, People's Republic of China

Tel +86055 I 63538435

Fax +86055163538435

Email zhouqiao1975@163.com
Abstract: Glioma is the most common type of primary brain tumor. Despite the combination of surgery, chemotherapy, and radiotherapy, the median survival duration of patients with malignant glioma is still very short. Temozolomide (TMZ) is the primary and most promising therapeutic drug for glioma; however, it is easy to develop acquired resistance during treatment. Activation of receptor tyrosine kinases (RTKs) has been identified to be involved in the acquisition of resistance toward many anticancer drugs. So inhibition of RTKs might be a promising therapeutic strategy for overcoming or attenuating acquired drug resistance. Here, we have investigated the anticancer activities of BMS-536924, an ATP-competitive IGF-1R/IR inhibitor in glioma, especially TMZ-resistant glioma, both in vitro and in vivo. We found that BMS-536924 could effectively reduce viability of both TMZ-sensitive and -resistant glioma cells. BMS-536924 induced dramatic apoptosis in TMZ-resistant cells, and it also dramatically inhibited migration of TMZ-resistant cells. Importantly, BMS-536924 significantly suppressed glioma tumor growth in vivo. This is the first report on anticancer activity of BMS-536924 in glioma. BMS-536924 is a promising compound in the therapy of glioma, especially of TMZresistant glioma, which might shed new light on glioma therapy.

Keywords: resistance, proliferation, motility, brain tumor resistance

\section{Introduction}

Glioma is the most common type of primary brain tumor and one of the most notorious of malignancies, and it accounts for more than $40 \%$ of all malignant primary brain tumors. Malignant gliomas, the most common primary malignant brain tumors, are aggressive, highly invasive, and neurologically destructive. Despite the combination of surgery, chemotherapy, and radiotherapy, the median survival duration of patients with malignant glioma is only $9-14$ months. ${ }^{1-5}$

Currently, the first-line postsurgical therapy for glioma patients is the combination of temozolomide (TMZ) and regional fractionated radiation, followed by TMZ alone. TMZ, a 3-methyl derivative of mitozolomide, is an alkylating chemotherapeutic drug that readily crosses the blood-brain barrier in glioma patients. It can efficiently reduce viability and induce apoptosis of glioma cells. ${ }^{6-8}$ Although TMZ is currently the most promising chemotherapy for glioma and the addition of TMZ to radiotherapy has resulted in an overall increase in survival of glioma patients, it is not always effective, and most therapies have eventually failed in glioma patients due to incomplete tumor resection and the apparent resistance of tumor cells to irradiation and TMZ. ${ }^{9-11}$ 
Some tumors are insensitive to $\mathrm{TMZ}$, while some tumors may develop acquired TMZ resistance during treatment. ${ }^{9-11}$ Therefore, TMZ resistance represents a major obstacle in the treatment of glioma, which needs to be resolved or attenuated.

Drug resistance arises with a wide variety of mechanisms. Both preclinical and clinical studies have found that a critical underlying mechanism for $\mathrm{TMZ}$ resistance development is the activation of receptor tyrosine kinases (RTKs). ${ }^{12-17}$ So inhibition of RTKs might provide a potential therapeutic strategy for glioma, especially TMZ-resistant glioma. Here we have investigated the anticancer activities of BMS536924, an ATP-competitive IGF-1R/IR inhibitor, in glioma, especially TMZ-resistant glioma, both in vitro and in vivo.

\section{Materials and methods Materials}

BMS-536924 was purchased from AdooQ ${ }^{\mathrm{TM}}$ BioScience (Irvine, CA, USA). TMZ and all other reagents were provided by Sigma-Aldrich Corp (St Louis, MO, USA).

\section{Cells and cell culture}

Human glioma cell lines M059K and U87MG were obtained from the American Type Culture Collection (Manassas, VA, USA). TMZ-resistant cell lines M059K-R and U87MG-R were obtained by culturing M059K or U87MG cells with gradually increased doses $(2 \sim 20 \mu \mathrm{M})$ of TMZ for 4 months. All the cells were cultured in Dulbecco's Modified Eagle's Medium (DMEM) medium with 10\% fetal bovine serum (FBS), 100 units $/ \mathrm{mL}$ penicillin, and $100 \mu \mathrm{g} / \mathrm{mL}$ streptomycin. Cells were maintained at $37^{\circ} \mathrm{C}$ in an atmosphere comprising $95 \%$ air and $5 \% \mathrm{CO}_{2}$.

\section{Cell viability assay}

TMZ-sensitive and -resistant glioma cells were plated in 96-well white plates at $5 \times 10^{3}$ cells/well, and BMS-536924 (12.5 800 nM) was added and incubated for 24, 48, or 72 hours. A CellTiter-Glo ${ }^{\circledR}$ kit (Promega Corp, Fitchburg, WI, USA) was used to assess cell viability. Briefly, a cell lysis/ ATP detection reagent was added $(30 \mu \mathrm{L} /$ well $)$, cells were shaken for 10 minutes, and the luminescence was examined using a spectrophotometer (Molecular Devices, Sunnyvale, $\mathrm{CA}, \mathrm{USA})$. All half-maximal inhibitory concentration $\left(\mathrm{IC}_{50}\right)$ values were determined using Compusyn software.

\section{Flow cytometry analysis}

TMZ-sensitive and -resistant cells were plated in six-well plates at a density of $5 \times 10^{5}$ cells/well and treated with
BMS-536924 (100 800 nM) for 48 hours. Then the cells were fixed with $70 \%$ ethanol and stained with propidium iodide (PI) in the presence of RNase $(1 \mathrm{~g} / \mathrm{L}), 1 \mathrm{~g} / \mathrm{L}$ sodium citrate, and $0.5 \%$ Triton $\mathrm{X}-100(\mathrm{v} / \mathrm{v})$, for 30 minutes in the dark. A FacsCalibur ${ }^{\mathrm{TM}}$ (BD Biosciences, Franklin Lakes, NJ, USA) sorter was used to analyze cell apoptosis. The percentage of hypodiploidy was taken as a measure of apoptosis and quantified using ModFIT LT ${ }^{\mathrm{TM}}$ software.

\section{Cell nuclei staining}

TMZ-sensitive and -resistant cells were seeded onto coverslips and incubated with BMS-536924 (100 800 nM) for 48 hours, then cells were fixed with $4 \%$ paraformaldehyde for 30 minutes and permeabilized with $0.1 \%$ Triton $\mathrm{X}-100$ for 20 minutes. After being blocked with 5\% normal goat serum for 30 minutes, the cells were stained with Hoechst 33342 for 15 minutes. Finally, cells were washed and imaged using a microscope (Nikon Corp, Tokyo, Japan).

\section{Cell migration assays}

Transwell assay

A Transwell ${ }^{\circledR}$ model was used to measure cell migration. TMZ-sensitive and -resistant cells were seeded in the upper compartments of Transwell 24-well Boyden chambers (Costar, Bedford, MA, USA) at a density of $5 \times 10^{4}$ cells/ well, in media without serum. Then, $600 \mu \mathrm{L}$ of serum-free media containing $10 \mu \mathrm{g} / \mathrm{mL}$ fibronectin were put in the lower compartments. After an incubation with BMS-536924 (10 160 nM) for 8 hours, cells were fixed and stained with $0.1 \%$ crystal violet. The cells that failed to migrate were removed, and those that migrated to the lower chamber were imaged under a microscope (Nikon Corp). Then the migrated cells were lysed with $10 \%$ acetic acid, and the absorbance was measured at $595 \mathrm{~nm}$.

\section{Wound healing assay}

TMZ-sensitive and -resistant cells were plated into 96-well plates at a density of $2 \times 10^{4}$ cells/well. After reaching confluence, cells were scratched using a pipette tip to generate a wound. Serum-free media with BMS-536924 (10 160 nM) were added and incubated for 8 hours. Then the cells were imaged under a microscope.

\section{Immunohistochemistry}

Tumor tissue samples were fixed in phosphate-buffered formalin and embedded in paraffin. Paraffin slices were then taken, at a thickness of $4 \mu \mathrm{m}$, before being mounted to slides. The slides were then deparaffinized in three 5-minute 
xylene washes before being rehydrated through the following series of alcohol washes: two washes of $100 \%$ ethanol for 10 minutes each, two washes of $95 \%$ ethanol for 10 minutes each, and then a final deionized water wash for 1 minute, with gentle agitation. Slides were incubated with $10 \mathrm{mM}$ sodium citrate buffer $(\mathrm{pH}$ 6.0) and heated for 1 hour. The slides were then washed three times in deionized water for 5 minutes per wash before a 30-minute block in 5\% normal goat serum. Terminal deoxynucleotidyl transferase dUTP nick end labeling (TUNEL) staining was performed using a Calbiochem FragEL ${ }^{\text {TM }}$ kit according to the manufacturer's instructions, followed by nuclei staining with 4',6-diamidino2-phenylindole (DAPI) for 15 minutes before imaging using fluorescent microscopy.

\section{Tumor growth suppression in vivo}

$\mathrm{Nud} /$ nud mice were purchased from the Shanghai Institute of Materia Medica, Chinese Academy of Sciences in Shanghai, People's Republic of China. All animal experiments were carried out in accordance with protocols approved by the Animal Care and Use Committees of the University of Science and Technology of China. M059K-R and M059K cells were injected subcutaneously into the axillary regions of selected nud/nud mice $\left(5 \times 10^{6}\right.$ cells $/ 100 \mu \mathrm{L} /$ mouse $)$. Cells were allowed to grow to reach a volume of $50 \mathrm{~mm}^{3}$ before the mice were randomized to the following experimental groups: BMS536924 (20 mg/kg), BMS-536924 (40 mg/kg), and control. Each group had seven animals. Every other day, tumors were measured with a microcaliper and the animals were weighed. Total tumor volumes were calculated as follows:

Tumor volume $\left(\mathrm{mm}^{3}\right)=$ width $\times$ width $\times$ length $\times 0.5$

Mice were euthanized 2 weeks later, and the tumors were collected and saved for later analysis.

\section{Statistics}

Both Student's $t$-test and analysis of variance (ANOVA) were performed using StatView (SAS Institute, Cary, NC, USA). $P<0.05$ was considered statistically significant. The data shown are the mean values of measures taken in triplicate. Error bars indicate standard deviation.

\section{Results}

\section{BMS-536924 decreases viability of both} TMZ-sensitive and -resistant glioma cells

$\mathrm{TMZ}$ is the primary and most promising therapeutic drug for glioma. In order to get TMZ-resistant cell lines, we cultured
$\mathrm{M} 059 \mathrm{~K}$ and U87MG cells with gradually increased doses $(2 \sim 20 \mu \mathrm{M})$ of TMZ for 4 months. Then we examined and compared the sensitivity of both TMZ-sensitive (M059K and U87MG) and -resistant (M059K-R and U87MG-R) cells toward TMZ. As shown in Figure $1 \mathrm{~A}$ and $\mathrm{B}$, there was a significant increase in $\mathrm{IC}_{50}$ for resistant cells compared with matched parental sensitive cells $(P<0.05)$.

IGF-1R and IR have been shown to play a critical role in promoting the cell viability of glioma cells. ${ }^{18-20}$ Moreover, RTKs, including IGF-1R/IR, are known to mediate the generation of tumor resistance. ${ }^{12-17}$ Therefore, we next examined the effect of BMS-536924 on the viability of both TMZ-sensitive and -resistant glioma cells. As shown in Figure 1C and D, BMS-536924 significantly reduced the viability of both $\mathrm{TMZ}$-sensitive and -resistant lines, in a dose-dependent manner $(P<0.05)$. Moreover, BMS-536924 also significantly reduced the viability of both TMZ-sensitive and -resistant lines, in a time-dependent manner (Figure 1E and F) $(P<0.05)$.

\section{BMS-536924 induces apoptosis of both TMZ-sensitive and -resistant glioma cells}

IGF-1R/IR-mediated signaling plays crucial roles in inhibiting apoptosis and increasing cell viability, ${ }^{18-20}$ so we next examined BMS-536924-induced apoptosis in both TMZsensitive and -resistant glioma cells. Figure 2A shows that the fraction of cells with subG1 DNA content significantly increased after treatment with BMS-536924 $(P<0.05)$. We also observed the nucleus morphology changes in both TMZ-sensitive and -resistant glioma cells after treatment with BMS-536924. Consistently, after treatment with BMS536924, the nuclei of both TMZ-sensitive and -resistant glioma cells exhibited a condensed and fragmented morphology (indicated with arrow heads), which is a characteristic for apoptosis (Figure 2B and C), suggesting that BMS-536924 could induce dramatic apoptosis in both TMZ-sensitive and -resistant glioma cells. These data are consistent with the viability suppression results showed above.

\section{BMS-536924 suppresses phosphorylation of IGF-IR and IR in TMZ-resistant glioma cells}

We observed the effect of BMS-536924 on phosphorylation of IGF-1R and IR in M059K-R and U87MG-R cells, using western blot assay. As shown in Figure 3, BMS-536924 significantly inhibited phosphorylation of IGF-1R and IR in these TMZ-resistant cells. In contrast, there were no significant changes in total protein levels (Figure 3 ). 
A

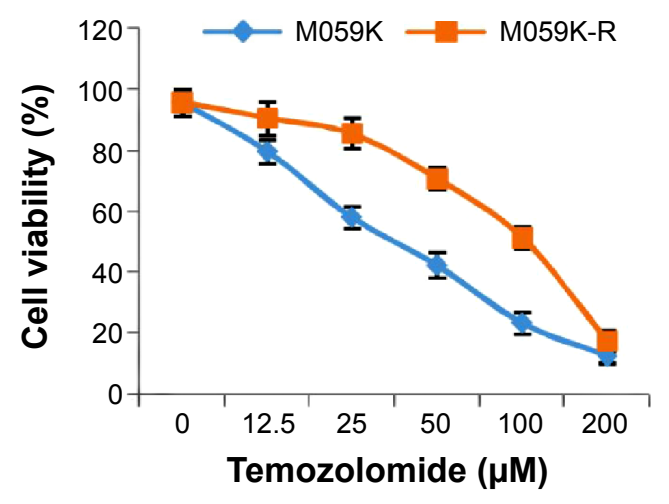

C

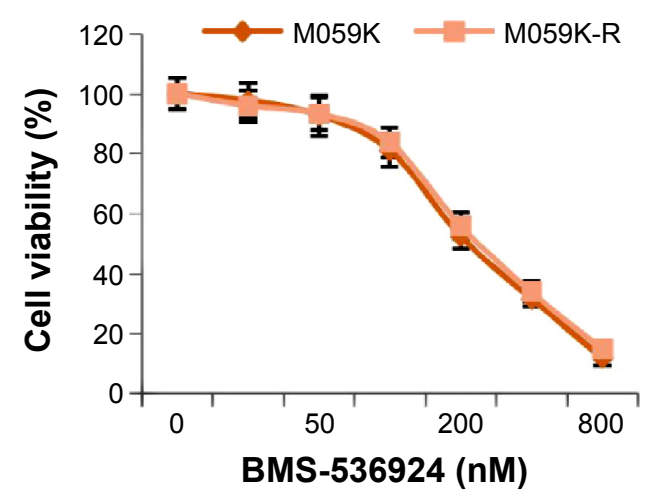

E

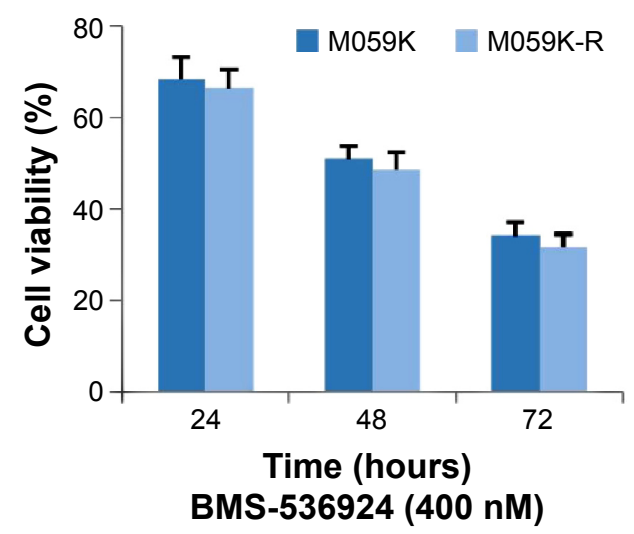

B

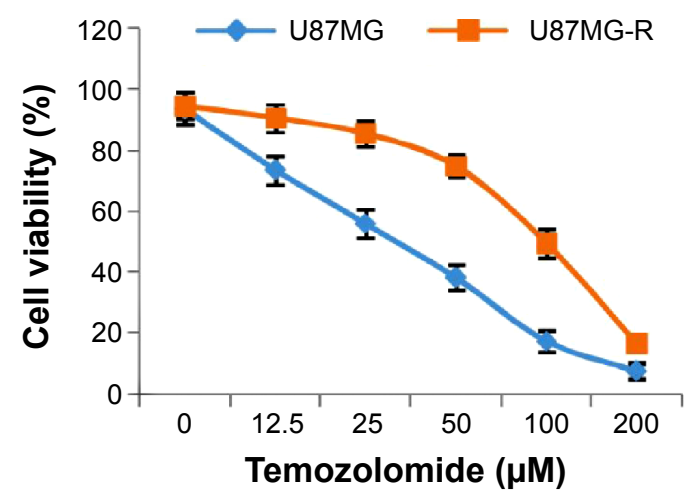

D

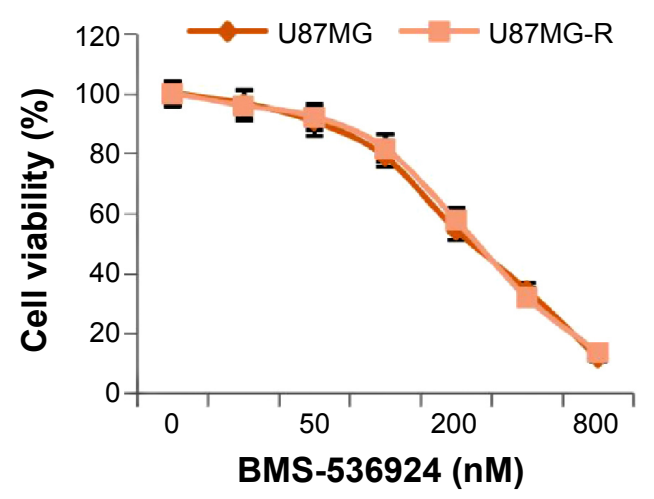

$\mathbf{F}$

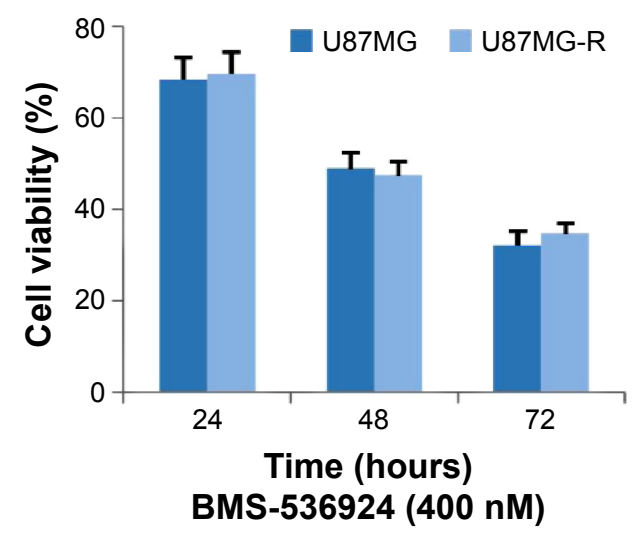

Figure I BMS-536924 reduces viability of both TMZ-sensitive and -resistant glioma cells.

Notes: (A and B) M059K and U87MG cells were induced to become TMZ-resistant cells (M059K-R and U87MG-R) by incubation with gradually increased doses (2 20 $\mu$ M) of TMZ for 4 months. The TMZ sensitivity was then examined using a cell viability assay. (C and D) Both TMZ-sensitive and -resistant cells were treated with BMS-536924 (I2.5 $800 \mathrm{nM}$ ) for 72 hours, followed by measurement of cell viability. (E and F) Both TMZ-sensitive and -resistant cells were treated with BMS-536924 (400 nM) for 24, 48 , or 72 hours, followed by measurement of cell viability.

Abbreviation: TMZ, temozolomide.

\section{BMS-536924 inhibits migration of both} TMZ-sensitive and -resistant glioma cells IGF-1R/IR-mediated signaling plays critical roles in the migration of cancer cells. ${ }^{21,22}$ So we next investigated the effect of BMS-536924 on migration of both TMZ-sensitive and -resistant glioma cells. Figure 4A and B shows that BMS-536924 inhibited migration of both TMZ-sensitive and -resistant glioma cells in a Transwell model $(P<0.05)$. Similar inhibition of cell migration was also observed in a wound healing model (Figure 4C and D) $(P<0.05)$. These data suggest that besides the suppression of viability, BMS-536924 could also suppress migration of both TMZ-sensitive and -resistant glioma cells. 
A

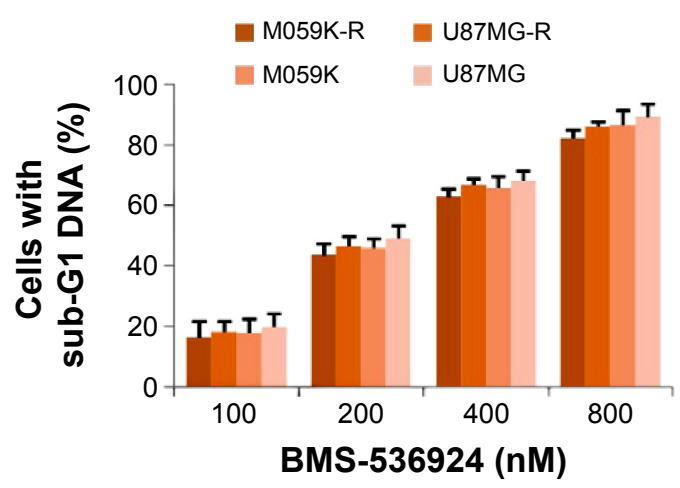

B

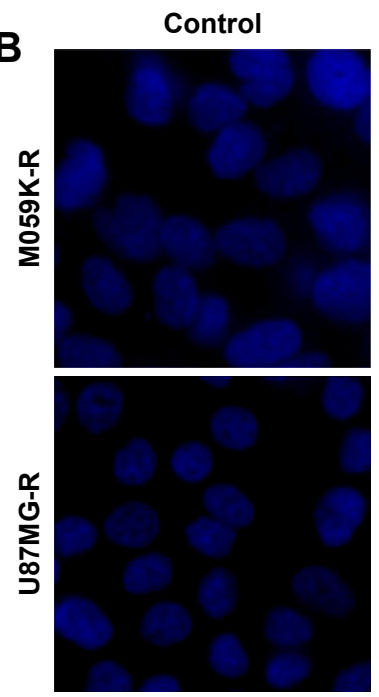

BMS-536924 (800 nM)

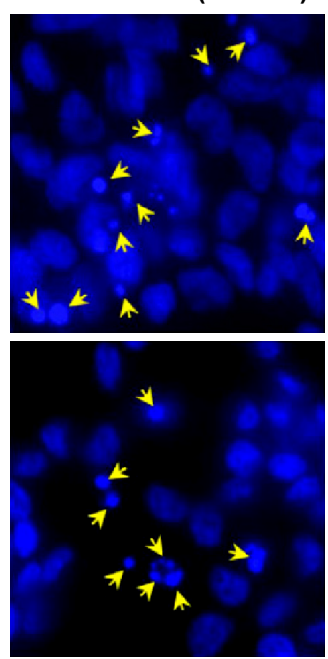

C

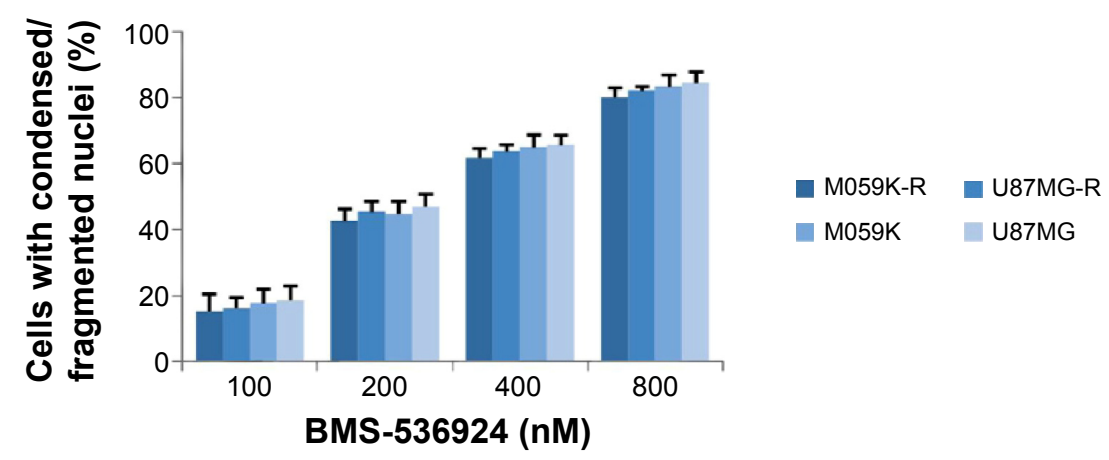

Figure 2 BMS-536924 induces apoptosis of both TMZ-sensitive and -resistant glioma cells.

Notes: (A) TMZ-resistant and -sensitive cells were treated with BMS-536924 (100 800 nM) for 48 hours, followed by PI staining and flow cytometry analysis. (B) TMZresistant and -sensitive cells were incubated with BMS-536924 (I00 800 nM) for 48 hours. The nuclei were stained with DAPI and analyzed using a fluorescent microscope. The representative images are shown (the fragmented nuclei were labeled by arrow heads). (C) The number of cells with condensed/fragmented nuclei was quantitated by counting in five random fields, and the inhibition was calculated.

Abbreviations: DAPI, 4',6-diamidino-2-phenylindole; PI, propidium iodide; TMZ, temozolomide.

\section{BMS-536924 suppresses both TMZ- sensitive and -resistant glioma tumor growth in vivo}

We next treated nud/nud mice with BMS-536924 (20 and $40 \mathrm{mg} / \mathrm{kg}$ ) and examined the effect of BMS-536924 (intraperitoneal injection) on M059K-R and M059K tumor growth in mice. The results show that the low dose of BMS-536924 $(20 \mathrm{mg} / \mathrm{kg})$ led to an intermediate level of tumor growth suppression, whereas the high dose of BMS-536924 $(40 \mathrm{mg} / \mathrm{kg})$ led to a significant inhibition on M059K-R and M059K tumor growth (Figure 5A and B) $(P<0.05)$. Consistent with the in vitro apoptosis data showed above, BMS-536924 induced dramatic apoptotic characteristics in tumors as examined using TUNEL, DAPI, and hematoxylin and eosin (H\&E) staining assays (Figure 5C). At the same time, administration of BMS-536924 was well-tolerated by healthy mice, without significant signs of overt toxicity on the main mice organs, including lung, liver, and kidney, or loss of weight $(P<0.05)$ (Figure 5D). Taken together, these results suggest that BMS-536924 could induce apoptosis and suppress the viability of TMZ-sensitive and -resistant glioma cells in vitro and suppress tumor growth in vivo.

\section{Discussion}

During recent decades, significant progress has been made in understanding cancer biology and in inventing new therapeutic strategies for cancers. Despite these achievements, we are still far from successfully managing cancers, and there is still a long way to go. Resistance to anticancer therapies, both intrinsic and acquired, constitutes one of the main causes of an inadequate response to treatment and diminished survival of cancer patients. ${ }^{23-25}$ Nowadays, research efforts 

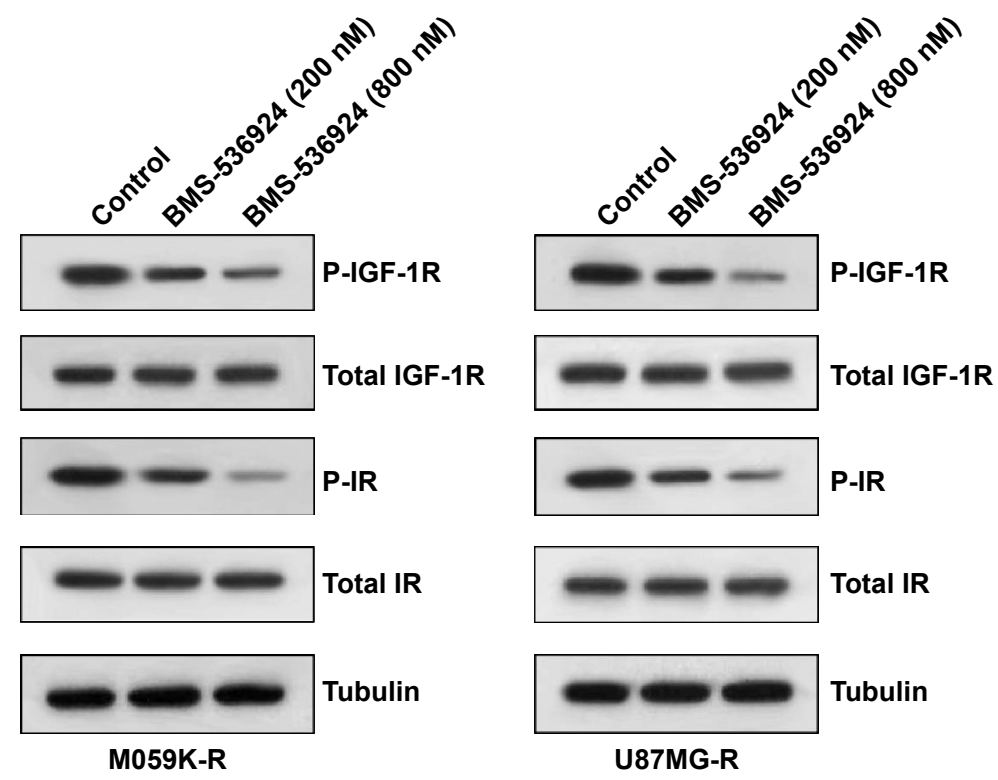

Figure 3 BMS-536924 suppresses phosphorylation of IGF-IR and IR in both TMZ-sensitive and -resistant glioma cells.

Notes: M059K-R and U87MG-R cells were seeded in six-well plates and incubated for 24 hours. Then the medium was replaced with serum-free medium, and BMS-536924 ( 200 and $800 \mathrm{nM}$ ) was added and incubated for 6 hours. Finally, the cells were stimulated with $30 \mathrm{ng} / \mathrm{mL}$ IGF-I or $3 \mu \mathrm{g} / \mathrm{mL}$ insulin for $30 \mathrm{minutes}$, then were lysed and analyzed by western blot, using the indicated antibodies.

A

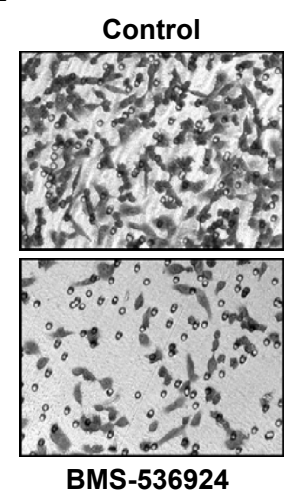

$(40 \mathrm{nM})$

C

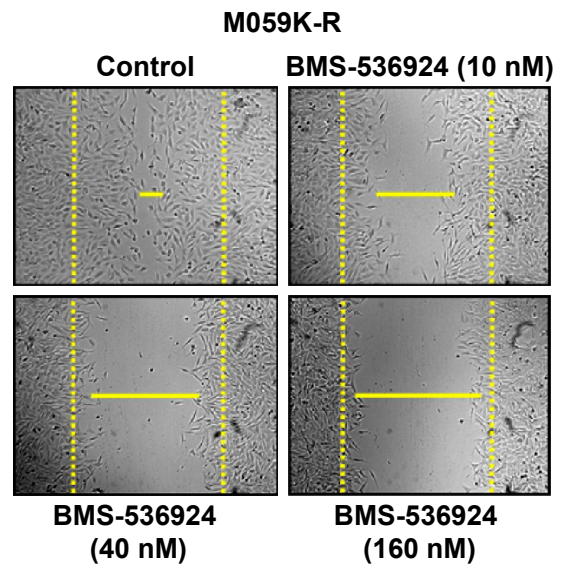

B

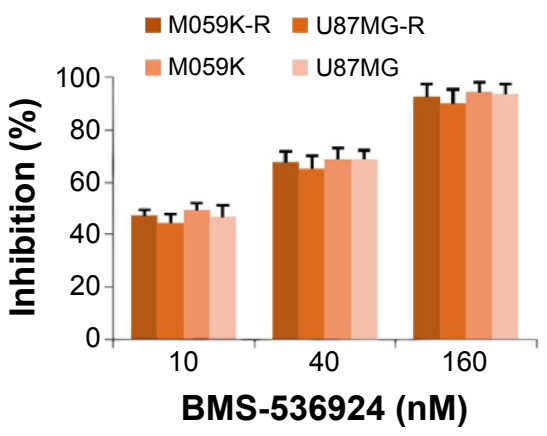

D

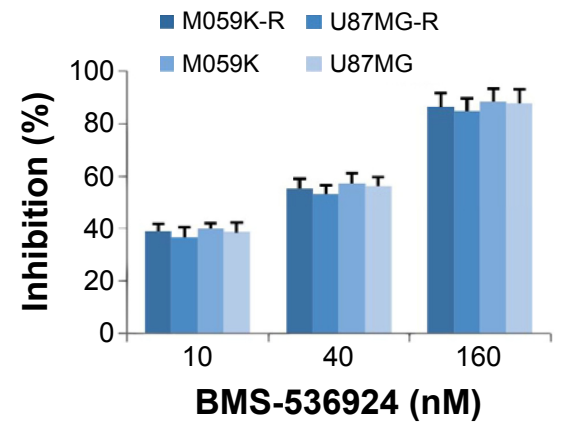

Figure 4 BMS-536924 inhibits migration of both TMZ-sensitive and -resistant glioma cells.

Notes: (A) TMZ-resistant and -sensitive cells were treated with BMS-536924 (10, 40, or $160 \mathrm{nM})$ for 8 hours. The nonmigrated cells on the upper surface of the filter were removed, and the migrated cells on the lower side were stained and photographed. The representative images are shown. Then, cells were lysed, and colorimetric determination was made at $595 \mathrm{~nm}$. (B) Quantitation of the inhibition from Transwell ${ }^{\circledR}$ assay. (C) A scratch was introduced into a monolayer of TMZ-resistant and -sensitive cells, followed by treatment with BMS-536924 (I0, 40, or $160 \mathrm{nM})$ for 8 hours. The width of the wounded cell monolayer was measured in five random fields, and representative images are shown. (D) Quantitation of the inhibition from the wound healing assay.

Abbreviation: TMZ, temozolomide. 
A

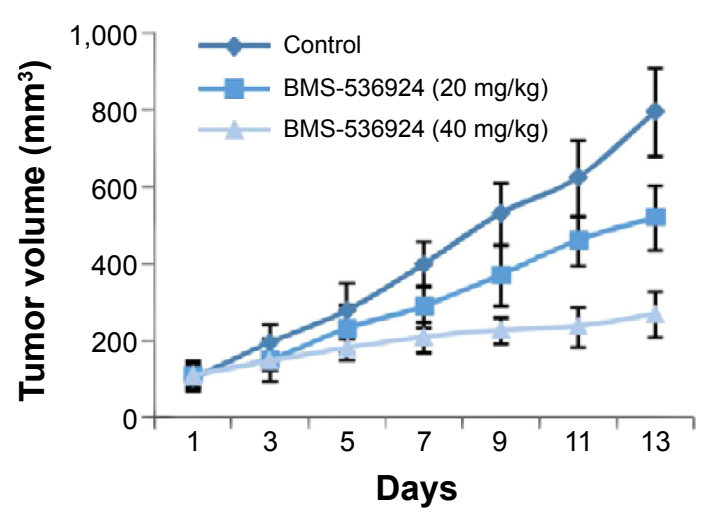

C
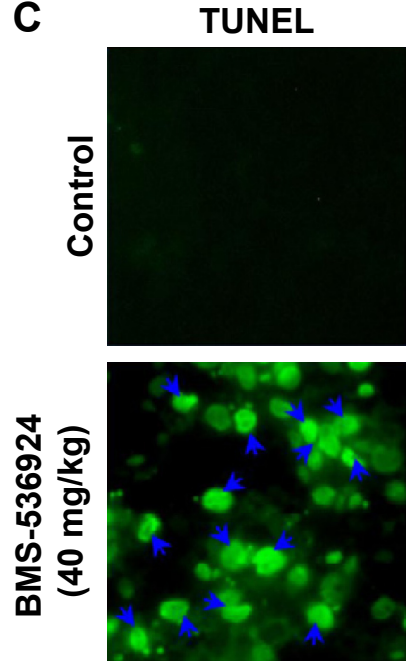

D

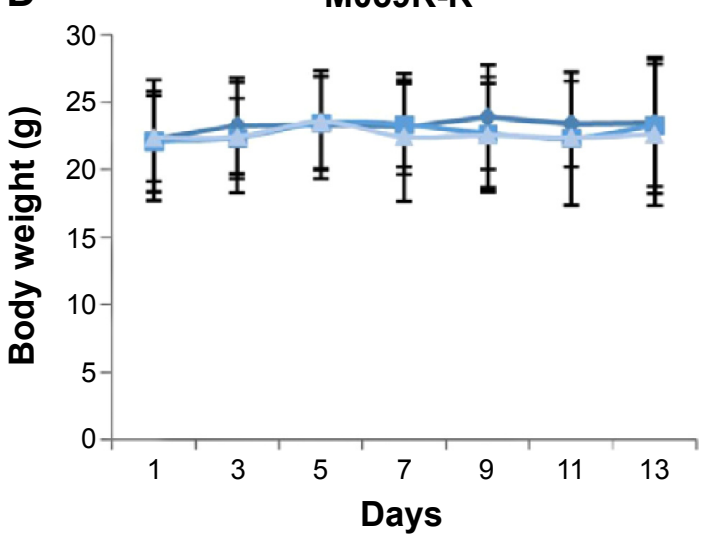

B

M059K

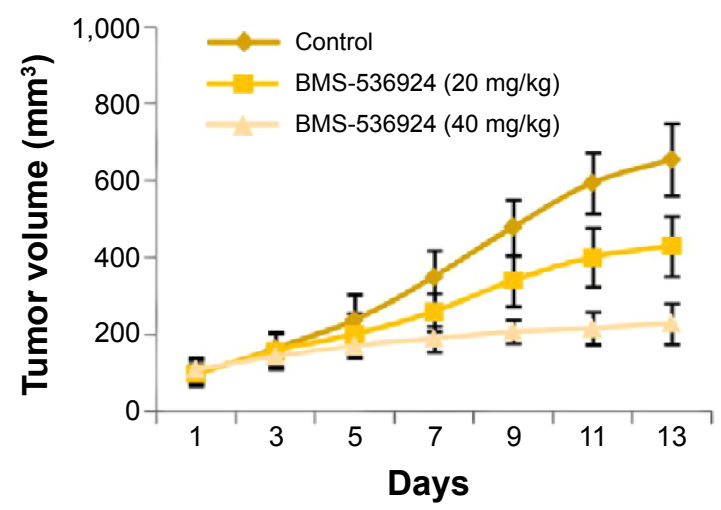

DAPI

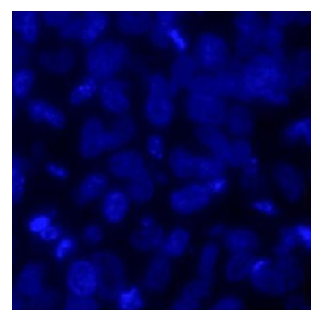

H\&E
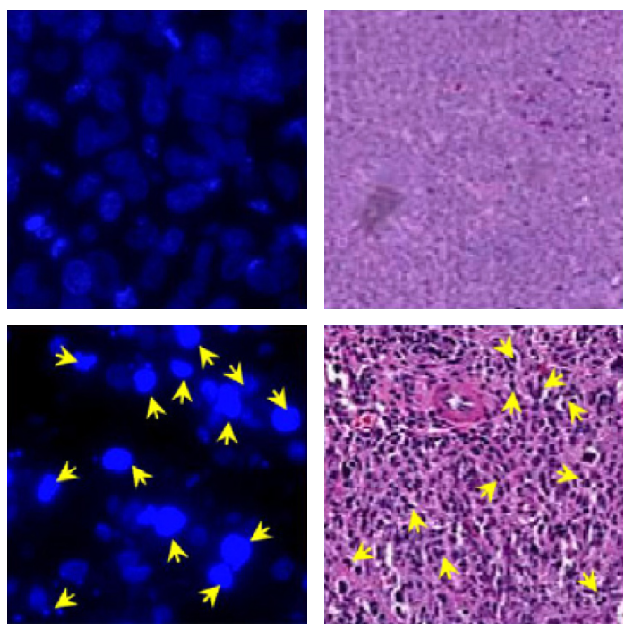

Control

- BMS-536924 (20 mg/kg)

BMS-536924 (40 mg/kg)

Figure 5 BMS-536924 suppresses M059K-R and M059K tumor growth in vivo.

Notes: (A and B) After inoculation of M059K-R and M059K cells, BMS-536924 (20 and $40 \mathrm{mg} / \mathrm{kg}$ ) was injected in mice every day. The tumors were measured every other day, and the tumor volumes are shown. (C) BMS-536924-induced apoptosis of M059K-R tumor cells in vivo was examined with TUNEL assay (green), DAPI staining (blue), or H\&E staining (purple); the apoptotic cells were labeled with arrowheads. (D) BMS-536924 had no significant cytotoxic effects on the body weight of mice during the treatments.

Abbreviations: DAPI, 4',6-diamidino-2-phenylindole; H\&E, hematoxylin and eosin; TUNEL, terminal deoxynucleotidyl transferase dUTP nick end labeling.

in cancer biology and pharmacology have been focused on developing therapeutic strategies to overcome resistance to anticancer drugs.

Glioma is well known as the most common primary aggressive malignant brain tumor of the central nervous system and has become one of the most lethal forms of cancer in human. ${ }^{1-5} \mathrm{TMZ}$ is the most commonly used chemotherapeutic drug in clinical trials for glioma. It seems that TMZ could work by sensitizing the tumor cells to radiation, and it has become the standard of care in glioma treatment, with 
a limited side-effect profile. ${ }^{6-8}$ However, a large number of patients are resistant to TMZ, which greatly compromises the clinical treatment. ${ }^{9-11}$ Thus, research on TMZ resistance is of great importance for ameliorating the therapeutic efficacy and alleviating the suffering of patients.

RTKs have been identified to be involved in the acquisition of resistance toward many anticancer drugs. ${ }^{12-17}$ Inhibition of RTKs might be a promising therapeutic strategy for overcoming or attenuating acquired drug resistance. It is imperative to explore new targets in the therapy of glioma, especially TMZ-resistant glioma. Considering the critical roles of IGF-1R/IR in tumor growth, metastasis as well as development of TMZ resistance, ${ }^{18-22}$ herein, we have investigated the anticancer activities of BMS-536924, an ATP-competitive IGF-1R/IR inhibitor, in glioma, especially TMZ-resistant glioma, both in vitro and in vivo. We found that BMS-536924 could effectively suppress viability of both TMZ-sensitive and -resistant glioma cells. Consistently, BMS-536924 induced significant apoptosis in both TMZ-sensitive and -resistant cells. In addition, BMS536924 also dramatically inhibited migration of both TMZsensitive and -resistant cells. Importantly, BMS-536924 significantly suppressed both M059K-R and M059K tumor growth in vivo. If BMS-536924 can be well used in clinical glioma therapy, it must pass through the blood-brain barrier first, which needs to be further confirmed. Moreover, our collaborators are developing more BMS-536924 analogues, including some smaller molecules that might more easily cross the blood-brain barrier. Some studies have reported that BMS-536924 had potential therapeutic effects on breast cancer, leukemia, and ovarian cancers. ${ }^{26-28}$ The effect of BMS-536924 on normal human cells also needs to be further confirmed. In our current animal model, administration of BMS-536924 did not exhibit any signs of overt toxicity in mice.

TMZ is the primary effective compound in glioma therapy. However, resistance to TMZ develops quickly, within only months. So overcoming or attenuating TMZ resistance becomes an urgent issue. The anticancer effect of BMS536924 on TMZ-resistant cells makes BMS-536924 or its analogues promising anticancer drug candidates for therapy of gliomas, especially of TMZ-resistant gliomas. This might shed new light on glioma therapy.

\section{Disclosure}

The authors report no conflicts of interest in this work.

\section{References}

1. Verhaak RG, Hoadley KA, Purdom E, et al; Cancer Genome Atlas Research Network. Integrated genomic analysis identifies clinically relevant subtypes of glioblastoma characterized by abnormalities in PDGFRA, IDH1, EGFR, and NF1. Cancer Cell. 2010;17(1): 98-110.

2. Lacroix M, Abi-Said D, Fourney DR, et al. A multivariate analysis of 416 patients with glioblastoma multiforme: prognosis, extent of resection, and survival. J Neurosurg. 2001;95(2):190-198.

3. Stupp R, Mason WP, van den Bent MJ, et al; European Organisation for Research and Treatment of Cancer Brain Tumor and Radiotherapy Groups; National Cancer Institute of Canada Clinical Trials Group. Radiotherapy plus concomitant and adjuvant temozolomide for glioblastoma. N Engl J Med. 2005;352(10):987-996.

4. Ostrom QT, Gittleman H, Farah P, et al. CBTRUS statistical report: Primary brain and central nervous system tumors diagnosed in the United States in 2006-2010. Neuro Oncol. 2013;15 Suppl 2:ii1-ii56.

5. Pretto F, Neri D. Pharmacotherapy of metastatic melanoma: emerging trends and opportunities for a cure. Pharmacol Ther. 2013; 139(3):405-411.

6. Athanassiou H, Synodinou M, Maragoudakis E, et al. Randomized phase II study of temozolomide and radiotherapy compared with radiotherapy alone in newly diagnosed glioblastoma multiforme. J Clin Oncol. 2005; 23(10):2372-2377.

7. Yung WK, Kyritsis AP, Gleason MJ, Levin VA. Treatment of recurrent malignant gliomas with high-dose 13-cis-retinoic acid. Clin Cancer Res. 1996;2(12):1931-1935.

8. Hegi ME, Diserens AC, Gorlia T, et al. MGMT gene silencing and benefit from temozolomide in glioblastoma. $N$ Engl J Med. 2005; 352(10):997-1003

9. Hegi ME, Diserens AC, Godard S, et al. Clinical trial substantiates the predictive value of O-6-methylguanine-DNA methyltransferase promoter methylation in glioblastoma patients treated with temozolomide. Clin Cancer Res. 2004;10(6):1871-1874.

10. Hegi ME, Liu L, Herman JG, et al. Correlation of O6-methylguanine methyltransferase (MGMT) promoter methylation with clinical outcomes in glioblastoma and clinical strategies to modulate MGMT activity. J Clin Oncol. 2008;26(25):4189-4199.

11. Adair JE, Johnston SK, Mrugala MM, et al. Gene therapy enhances chemotherapy tolerance and efficacy in glioblastoma patients. J Clin Invest. 2014;124(9):4082-4092.

12. Hauschild A, Agarwala SS, Trefzer U, et al. Results of a phase III, randomized, placebo-controlled study of sorafenib in combination with carboplatin and paclitaxel as second-line treatment in patients with unresectable stage III or stage IV melanoma. J Clin Oncol. 2009; 27(17):2823-2830.

13. Tentori L, Lacal PM, Graziani G. Challenging resistance mechanisms to therapies for metastatic melanoma. Trends Pharmacol Sci. 2013; 34(12):656-666.

14. Hartsough E, Shao Y, Aplin AE. Resistance to RAF inhibitors revisited. J Invest Dermatol. 2014;134(2):319-325.

15. Das Thakur M, Stuart DD. The evolution of melanoma resistance reveals therapeutic opportunities. Cancer Res. 2013;73(20):6106-6110.

16. Nazarian R, Shi H, Wang Q, et al. Melanomas acquire resistance to B-RAF(V600E) inhibition by RTK or N-RAS upregulation. Nature. 2010;468(7326):973-977.

17. Villanueva J, Vultur A, Lee JT, et al. Acquired resistance to BRAF inhibitors mediated by a RAF kinase switch in melanoma can be overcome by cotargeting MEK and IGF-1R/PI3K. Cancer Cell. 2010;18(6): 683-695.

18. Dayyani F, Parikh NU, Varkaris AS, et al. Combined Inhibition of IGF-1R/IR and Src family kinases enhances antitumor effects in prostate cancer by decreasing activated survival pathways. PLoS One. 2012;7(12): e51189. 
19. Buck E, Gokhale PC, Koujak S, et al. Compensatory insulin receptor (IR) activation on inhibition of insulin-like growth factor-1 receptor (IGF-1R): rationale for cotargeting IGF-1R and IR in cancer. $\mathrm{Mol}$ Cancer Ther. 2010;9(10):2652-2664.

20. Brodt P, Samani A, Navab R. Inhibition of the type I insulin-like growth factor receptor expression and signaling: novel strategies for antimetastatic therapy. Biochem Pharmacol. 2000;60(8):1101-1107.

21. Mancini M, Gariboldi MB, Taiana E, et al. Co-targeting the IGF system and HIF-1 inhibits migration and invasion by (triple-negative) breast cancer cells. Br J Cancer. 2014;110(12):2865-2873.

22. Subramani R, Lopez-Valdez R, Arumugam A, Nandy S, Boopalan T, Lakshmanaswamy R. Targeting insulin-like growth factor 1 receptor inhibits pancreatic cancer growth and metastasis. PLoS One. 2014; 9(5):e97016

23. Lippert TH, Ruoff HJ, Volm M. Current status of methods to assess cancer drug resistance. Int J Med Sci. 2011;8(3):245-253.

24. Liu FS. Mechanisms of chemotherapeutic drug resistance in cancer therapy - a quick review. Taiwan J Obstet Gynecol. 2009;48(3): 239-244.
25. Gottesman MM. Mechanisms of cancer drug resistance. Annu Rev Med. 2002;53:615-627.

26. Dool CJ, Mashhedi H, Zakikhani M, et al. IGF1/insulin receptor kinase inhibition by BMS-536924 is better tolerated than alloxan-induced hypoinsulinemia and more effective than metformin in the treatment of experimental insulin-responsive breast cancer. Endocr Relat Cancer. 2011;18(6):699-709.

27. Wahner Hendrickson AE, Haluska P, Schneider PA, et al. Expression of insulin receptor isoform $\mathrm{A}$ and insulin-like growth factor-1 receptor in human acute myelogenous leukemia: effect of the dual-receptor inhibitor BMS-536924 in vitro. Cancer Res. 2009;69(19):7635-7643.

28. Beauchamp MC, Knafo A, Yasmeen A, et al. BMS-536924 sensitizes human epithelial ovarian cancer cells to the PARP inhibitor, 3-aminobenzamide. Gynecol Oncol. 2009;115(2):193-198.

\section{Publish your work in this journal}

OncoTargets and Therapy is an international, peer-reviewed, open access journal focusing on the pathological basis of all cancers, potential targets for therapy and treatment protocols employed to improve the management of cancer patients. The journal also focuses on the impact of management programs and new therapeutic agents and protocols on

\section{Dovepress}

patient perspectives such as quality of life, adherence and satisfaction. The manuscript management system is completely online and includes a very quick and fair peer-review system, which is all easy to use. Visit http://www.dovepress.com/testimonials.php to read real quotes from published authors. 